\title{
Anabases
}

ANABASES Traditions et réceptions de l'Antiquité

$8 \mid 2008$

Varia

\section{Laurent PERNOT, L'Ombre du Tigre. Recherches sur la réception de Démosthène}

Pascal Payen

\section{OpenEdition}

Journals

Édition électronique

URL : http://journals.openedition.org/anabases/260

DOI : 10.4000/anabases.260

ISSN : 2256-9421

Éditeur

E.R.A.S.M.E.

Édition imprimée

Date de publication : 1 octobre 2008

Pagination : 298-300

ISSN : 1774-4296

Référence électronique

Pascal Payen, «Laurent PERNOT, L'Ombre du Tigre. Recherches sur la réception de Démosthène », Anabases [En ligne], 8| 2008, mis en ligne le 01 juillet 2011, consulté le 22 septembre 2020. URL : http:// journals.openedition.org/anabases/260 ; DOI : https://doi.org/10.4000/anabases.260

Ce document a été généré automatiquement le 22 septembre 2020.

(c) Anabases 


\title{
Laurent PERNOT, L'Ombre du Tigre. Recherches sur la réception de Démosthène
}

\author{
Pascal Payen
}

\section{RÉFÉRENCE}

Laurent PERNOT, L'Ombre du Tigre. Recherches sur la réception de Démosthène, Napoli,

M. D'Auria Editore, 2006, $355 \mathrm{p}$.

80 euros / ISBN 88-7092-260-X.

Dans la conclusion de ce livre de science et de conviction, Laurent Pernot définit avec acuité le sens que devrait prendre toute étude portant sur la réception de l'Antiquité : «La manière dont une œuvre antique a été lue et transmise ne se ramène pas à une suite d'épiphénomènes qu'il conviendrait de négliger pour se borner à un dialogue orgueilleux entre le texte originel et la science d'aujourd'hui. Il s'agit au contraire d'une dimension importante, qui pose "l'historicité de la compréhension" (Geschichtlichkeit des Verstehens) et engage l'"histoire de l'efficience" (Wirkungsgeschichte), selon les concepts, ici très pertinents, du philosophe H.-G. Gadamer. L'inscription dans le temps est un élément essentiel de la vie des œuvres. Le travail de l'histoire dans le texte participe à la construction du sens » (p. 302). L'histoire de la réception fait partie du sens que prennent, pour les Modernes, une œuvre, une institution, un usage, un genre littéraire, un corpus philosophique venus de l'Antiquité. Nous n'entretenons pas une relation directe avec les Anciens. Leurs héritages nous sont accessibles à travers des choix opérés souvent dès l'Antiquité elle-même; nous lisons les œuvres dans des éditions établies à partir de manuscrits dont les plus anciens, souvent du IX $\mathrm{X}^{\mathrm{e}}$ ou du $\mathrm{X}^{\mathrm{e}}$ siècles, sont eux-mêmes fort éloignés des œuvres originales, à travers un ensemble de relais; les corpus documentaires plus récents, qu'utilisent les historiens, ont été constitués à partir de champs de ruines et selon des méthodes sur 
lesquelles les savants modernes ne s'accordent pas toujours. Enfin, dans l'arc chronologique qui nous sépare des sociétés anciennes se déploie un ensemble de relations à l'Antiquité, en fonction des multiples modernités et contextes qui nous en séparent. Intégrer l'histoire de la réception à notre connaissance des mondes anciens renforce la distance critique qui conduit à mieux comprendre cet héritage, selon un double registre : pour lui-même et dans le rapport qu'il n'a cessé d'entretenir avec les temps et les sociétés qui se le sont approprié. Or l'histoire de la tradition n'est pas l'histoire d'une erreur. Elle doit, à ce titre, faire l'objet de la même analyse historique et critique que les productions des Anciens et être intégrée à elles.

Toute la complexité de la tradition interprétative relative à Démosthène se retrouve dès l'abord du livre, lorsque L. Pernot rappelle les différents sens qui s'attachent à la réputation de Démosthène comme thèrion (Anpíov), «bête ", mais en quel sens - «bête de scène ", "monstre sacré ", "lion ", " fauve ", « animal ", "salle bête »? -, depuis son adversaire politique, l'orateur Eschine, le premier à lui avoir donné ce nom, jusqu'à Clemenceau, à qui le rôle de défenseur de la patrie a valu le surnom de «Tigre » et qui s'est lui-même identifié à l'orateur athénien dans un Démosthène paru en 1926. Par rapport aux ouvrages et études existant sur la réception de Démosthène, l'objet de ce livre n'est pas de proposer une synthèse et une histoire continue. Ces "recherches " ont pour but de repérer les "bizarreries", les "anomalies", des points qui apparaissent "comme des nœuds de compréhension nouvelle» et des «champs d'expérimentation méthodologique». Quatre problèmes sont ainsi présentés et analysés, qui, plus que les éloges et les moments de gloire, concernent « les recyclages, les récupérations et les contestations de la légende démosthénienne ». Pour chacun d'entre eux, l'auteur a réuni le dossier le plus exhaustif possible des Testimonia, parmi les sources antiques et byzantines. Tous sont rassemblés, classés et traduits avec une rigueur exemplaire dans le chapitre $\mathrm{V}$ et dernier.

3 Le premier chapitre est consacré à une anecdote biographique, selon laquelle «le prince de tous les orateurs de la Grèce » (Quintilien) aurait été l'élève (l'« auditeur ») de Platon, ainsi que son "admirateur» et son "imitateur». Trente-sept testimonia l'attestent, depuis le $\mathrm{III}^{\mathrm{e}}$ siècle avant notre ère jusqu'à la Souda, au $\mathrm{x}^{\mathrm{e}}$ siècle, en passant par Cicéron, très attaché à fixer cette tradition, Quintilien, Tacite, Aulu-Gelle, Lucien, Diogène-Laërce, les néoplatoniciens et de nombreux commentateurs anonymes. La méthode qu'illustre L. Pernot, pour conduire l'analyse de ce dossier est exemplaire. La prise en compte de toute la tradition critique moderne, très partagée (même si le scepticisme l'emporte), et du contexte ancien montre qu'il n'existe pas d'obstacle chronologique à la rencontre de Platon et Démosthène en tant que maitre et élève. Mais la confrontation de leurs œuvres conduit plutôt à "être sensible à tout ce qui les sépare ». Dès lors, plutôt que de s'enfermer " dans une problématique positiviste ", qui ne permettra jamais de trancher avec certitude un point d'ordre factuel, il faut prendre acte de cette puissante tradition et se demander pourquoi «l'Antiquité y a cru et quel sens elle lui a prêté ». La biographie de Démosthène, à travers cette anecdote, devient un moyen d'analyser les rapports entre philosophie et rhétorique, et le thème de la rencontre avec Platon ne doit être considéré ni comme un simple renseignement, selon l'approche positiviste, ni comme un topos sans consistance. L'anecdote illustre bien plutôt un idéal de complémentarité et un rapport hiérarchique entre philosophie et rhétorique, et le récit biographique tient lieu, lorsqu'il est évoqué, d'ultime garantie de cette théorie. 
4 Le deuxième dossier concerne le problème de l'«engagement politique " de Démosthène, ou encore, selon les termes des Anciens, celui des rapports entre la parole et l'action. L. Pernot analyse une tradition qui remet en cause l'idéal du patriote engagé au service de la démocratie, de l'indépendance de sa cité et de la liberté des Grecs. De cette légende inversée, il privilégie trois moments. Dans le contexte d'éloge de la Seconde sophistique, il y eut aussi des voix discordantes (Plutarque, Lucien, Pausanias, Aelius Aristide, Athénée, Philostrate) qui témoignent de la vitalité de l'hellénisme, nullement enfermé dans la célébration béate du passé classique. Ainsi les réserves de Plutarque, dans la Vie de Démosthène, s'expliquent-elles notamment par le fait qu'il s'agit pour lui moins de célébrer Athènes seule que de permettre à toutes les cités de trouver leur place dans d'Empire romain. Un second moment où est discutée l'utilité du modèle politique démosthénien est le temps des Paléologues, à Byzance, lorsque Théodore Métochite (1270-1332) rédige, vers 1330-1331, un Essai sur Démosthène et Aelius Aristide (publié seulement en 1965). Selon lui, dans l'analyse convaincante que propose L. Pernot, Démosthène reste un modèle insurpassable du point de vue de la technique rhétorique, mais l'expérience politique qu'il a connue ne correspond plus aux exigences $\mathrm{du}$ présent. Le troisième temps de remise en cause du modèle démosthénien vient avec les ouvrages d'Engelbert Drerup (1871-1942), qui s'en prend à la «république des avocats ", c'est-à-dire à la France assimilée à l'Athènes démocratique de Démosthène, et lui oppose l'Allemagne impériale, miroir de la monarchie macédonienne. Dans le pamphlet de 1916, Aus einer alten Advokatenrepublik (Demosthenes und seine Zeit) - qu'il qualifie de «Kriegsbuch»-, comme dans l'ouvrage scientifique de 1923, Demosthenes im Urteile des Altertums, le parti pris est le même, qui se traduit jusque dans de quasi silence concernant l'histoire de la réception de Démosthène. Tout autre est le point de vue adopté par Clemenceau, dont le Démosthène (1926) se présente comme un plaidoyer pro domo d'autant plus paradoxal que l'Athénien est le vaincu de Chéronée (338), alors que Clemenceau est le vainqueur de 1918.

5 Le troisième chapitre est consacré à l'analyse de la fortune de la phrase d'Aelius Aristide où, dans son traité Pour les Quatre (apologie des quatre rhètores de l'Antiquité : Miltiade, Thémistocle, Cimon et Périclès, que Platon avait critiqués dans le Gorgias), il qualifie Démosthène d'«empreinte de quelque Hermès Logios descendue parmi les hommes ». Hermès Logios préside à la parole et figure à ce titre comme patron des orateurs. L'expression « empreinte d'Hermès Logios » a connu une grande fortune, du $\mathrm{IV}^{\mathrm{e}}$ au XIV siècle, et a été l'objet de nombreux « réemplois et recyclages ", qui attestent presque tous que les enjeux concernent moins la figure de Démosthène lui-même, que les fonctions reconnues à la rhétorique comme discipline porteuse des valeurs de la paideia, moyen d'action politique, rivale de la philosophie, et même comme «modèle culturel doté d'une dimension religieuse ».

6 Dans le quatrième chapitre, l'analyse est concentrée également sur un bref passage, emprunté au discours Sur la couronne : le serment que prête Démosthène en rappelant le souvenir des combattants des guerres Médiques ( $\$ 208$ ), pour justifier sa politique d'alliance avec Thèbes, bien qu'elle ait conduit à la défaite de Chéronée. Contre le Perse ou contre Philippe, c'est la même générosité au service de tous les Grecs qui inspire les Athéniens. L. Pernot étudie tout le dossier des quarante-trois passages antiques et byzantins relatifs à ce serment, en prêtant un soin extrême aux différents contextes qui modifient le sens du texte et qui renseignent sur la fonction de la référence à Démosthène : contexte culturel, y compris linguistique (état du grec, fonction du latin), 
contexte politique, avec la domination romaine par exemple, contexte religieux avec l'instauration du christianisme, chez les Pères de l'Église. L'enquête est prolongée, mais plus rapidement, à la Renaissance (Leonardo Bruni, Pierre Danès), puis de Jaucourt à Marmontel, de Chateaubriand à Paul-Louis Courrier (voir les p. 117-130).

7 La réception d'un auteur ancien - ce livre le montre à chaque page - n'est jamais passive. Analyse philologique et enquête historique se croisent et se renforcent, pour apporter ici les preuves que « les usages qui ont été faits de Démosthène [...] montre[nt] une circulation constante entre des domaines qu'on aurait tort de croire séparés : entre monde grec et monde latin, entre rhétorique et philosophie, entre paganisme et christianisme, entre l'Antiquité et Byzance, entre l'érudition ancienne et les savoirs modernes » (p. 303). Par la richesse et l'implacable rigueur de ses analyses, et par sa facture même (avec le dossier complet des Testimonia et ses cinq indices), ce livre est appelé à devenir lui-même un classique des études de réception et un instrument de travail indispensable.

\section{AUTEURS}

\section{PASCAL PAYEN}

Université de Toulouse (UTM)

payen@univ-tlse2.fr 